Received: 2020-03-26

Accepted: 2020-06-03

Published: 2020-11-30

\section{Seroma formation After Spine SURGery Found 17 Months Postoperatively}

\author{
Jamal Hasoon, $\mathrm{MD}^{1}$ \\ Amnon A Berger, MD, PhD ${ }^{1}$ \\ Ivan Urits, MD ${ }^{1}$ \\ Vwaire Orhurhu, MD² \\ Omar Viswanath, $\mathrm{MD}^{3-5}$ \\ Alaa Abd-Elsayed, MD ${ }^{6}$
}

Background: A seroma is a collection of serous fluid that commonly occurs as a postoperative complication. Most seromas are diagnosed several days after surgery and typically resorb over a few months. While some seromas are asymptomatic, others can cause significant complications. Most seromas are easily treatable when the site is readily accessible. However, seroma formation after spine surgery can be more challenging to diagnose and manage due to anatomic considerations. Seroma formation following spine surgery is considered a rare postoperative complication, but has been recognized as a risk for rehospitalization and instrumentation.

\title{
Case
}

Presentation: We describe a 72-year-old woman, who underwent spine surgery 17 months prior, that developed recurrence of her back pain and radiculopathy after a mechanical fall. She underwent magnetic resonance imaging, which demonstrated a large $7.5 \times 8 \times 4.5-\mathrm{cm}$ seroma around the site of her prior surgery. She was started on neuropathic pain medications and referred to a neurosurgeon for further evaluation as the location of the seroma interfered with the ability to treat her with most interventional pain procedures.

Conclusion: This case illustrates that seromas can recur as a postoperative complication after spine surgery, distant from the original procedure. Additionally, this case reinforces the importance of reimaging patients when there is a history of trauma before considering routine interventional pain procedures.

Key words: Lumbar radiculopathy, spine surgery, chronic pain, complications

\footnotetext{
From: ' ${ }^{B}$ eth Israel Deaconess Medical Center, Department of Anesthesia, Critical Care, and Pain Medicine, Harvard Medical School, Boston, MA; ${ }^{2}$ Massachusetts General Hospital, Department of Anesthesia, Critical Care, and Pain Medicine, Harvard Medical School, Boston, MA; ${ }^{3}$ Valley Anesthesiology and Pain Consultants - Envision Physician Services, Phoenix, AZ; ${ }^{4}$ Department of Anesthesiology, University of Arizona College of Medicine, Phoenix, AZ; ${ }^{5}$ Department of Anesthesiology, Creighton University School of Medicine, Omaha, NE; ${ }^{6}$ Department of Anesthesiology, University of Wisconsin School of Medicine and Public Health, Madison, WI

Corresponding Author: Jamal Hasoon, MD E-mail: jhasoon@bidmc.harvard.edu Disclaimer: There was no external funding in the preparation of this manuscript.

Conflict of interest: Each author certifies that he or she, or a member of his or her immediate family, has no commercial association (i.e., consultancies, stock ownership, equity interest, patent/licensing arrangements, etc.) that might pose a conflict of interest in connection with the submitted manuscript.
} 


\section{BACKGROUND}

A seroma is a collection of serous fluid that commonly occurs as a postoperative complication, especially with abdominal surgery. Inflammation as part of the woundhealing process can create an exudate between tissue layers which can subsequently lead to a seroma formation. Most seromas are diagnosed several days after surgery and typically resorb over a few months. While some seromas are asymptomatic, others can cause pain, swelling, drainage, and could be complicated further with infections. Seromas often resolve with expectant management when small, but can require invasive management with incision and drainage if they become too large. Most seromas are easily treatable when the site is readily accessible. However, seroma formation after spine surgery can be more challenging to diagnose and manage due to anatomic considerations.

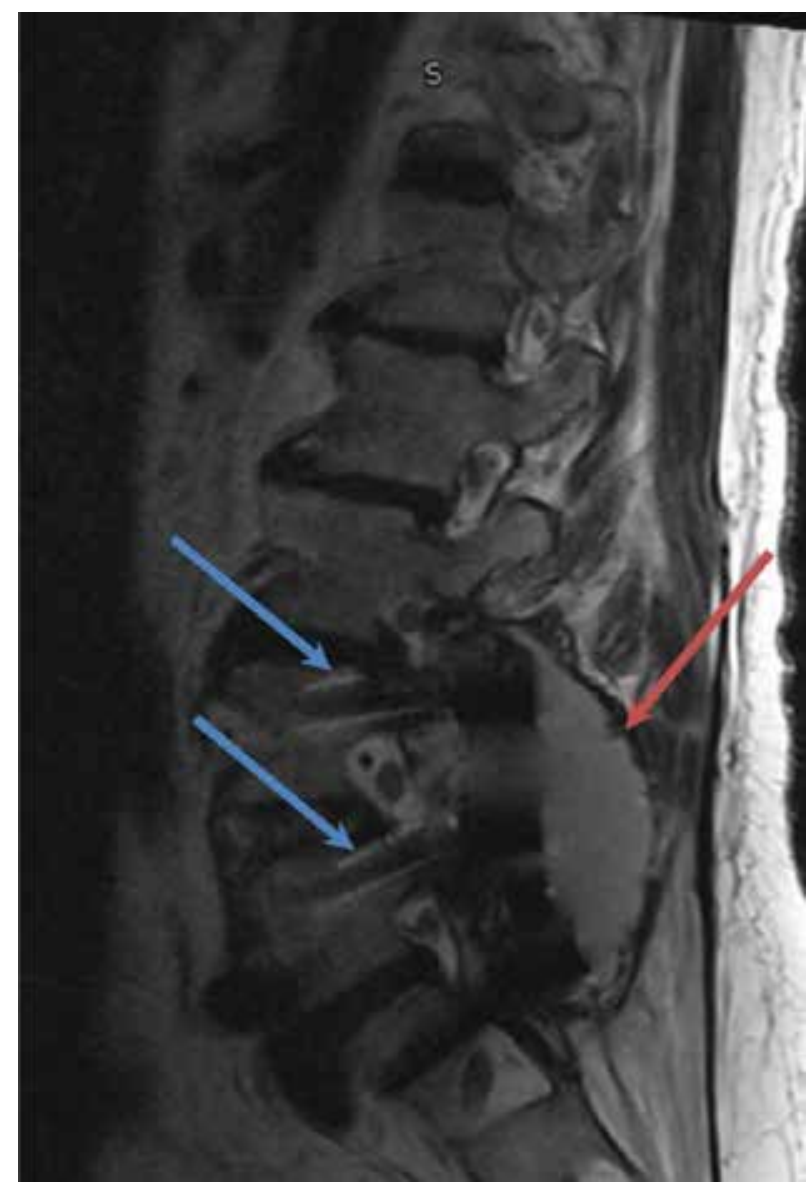

Fig. 1. Sagittal lumbar MRI. MRI image clearly demonstrates hardware left from the patient's previous spinal surgery (blue arrows) and seroma (red arrow).
Spine surgeries in the United States have been on the rise and have gone up $220 \%$ between 1990 and 2000. Unfortunately, the rate of complications has also risen proportionally (1). Seroma formation following spine surgery is considered a rare postoperative complication but has been recognized as a risk for rehospitalization and instrumentation (2). Risk factors for the development of seromas after spine surgery include the normal risks of seroma formation, with the possibility of recombinant humanized bone morphogenic protein-2 (rhBMP-2) as another potential risk factor. A recent article demonstrated a $1.22 \%$ complication rate with the use of rhBMP-2, an odds ratio of 1.89 compared to nonutilization (3). However, other reports have demonstrated that postoperative seromas can occur after spine surgery regardless of the use of rhBMP-2 (4). As with other surgeries, obesity also significantly increases the risk of seroma formation after spine surgery (5). Again, the diagnosis of a seroma is more difficult after spine surgery since it is harder to visualize and access.

\section{CASE PRESENTATION}

Here we present the case of a 72-year-old woman who underwent a L4-S1 anterior and posterior spinal fusion 17 months prior. She presented to the pain clinic with low back pain and radiculopathy following a mechanical fall. She initially had positive results after her spinal fusion, but slowly began to experience recurrence of her back pain with radiculopathy along the L5-S1 dermatomes bilaterally. She described her pain intensity as 3 out of 10 at rest that increased to 8 out of 10 with activity. She demonstrated a positive straight leg test bilaterally. The patient underwent imaging with lumbar spine magnetic resonance imaging (MRI), which demonstrated a large, nonenhancing, $7.5 \times 8 \times 4.5-\mathrm{cm}$ fluid collection in the posterior paraspinal soft tissue along the hardware left from her previous back surgery; this was consistent with seroma formation. MRI findings also included clumping of the cauda suggestive of possible arachnoiditis (Figs. 1 and 2). Given the complexity of this patient's anatomy due to the seroma, the patient was started on neuropathic pain medications and referred to a neurosurgeon for further evaluation of her symptoms before considering interventional pain injections. Unfortunately, the patient was lost to follow-up after the referral and did not keep her appointment with our neurosurgical team. 


\section{DISCUSSION}

Much like any space-occupying lesion, a seroma can result in nerve root compression causing symptoms of pain and neurological deficits. Additionally, arachnoiditis could also lead to neurologic symptoms such as pain and radiculopathy. Arachnoiditis is an inflammatory condition of the arachnoid mater around the central nervous system, including the brain and spinal cord. latrogenic causes of arachnoiditis can occur from surgery as well as intrathecal injection of medications, including steroids.

\section{CONCLUSION}

To our knowledge, this is the first report of a spine seroma formation more than 17 months postoperatively following trauma. This case illustrates a few educational points that are highlighted in this brief article. First, seromas can recur as a postoperative complication after spine surgery distant from the original procedure. Additionally, it is important to consider the possibility of iatrogenic arachnoiditis in patients

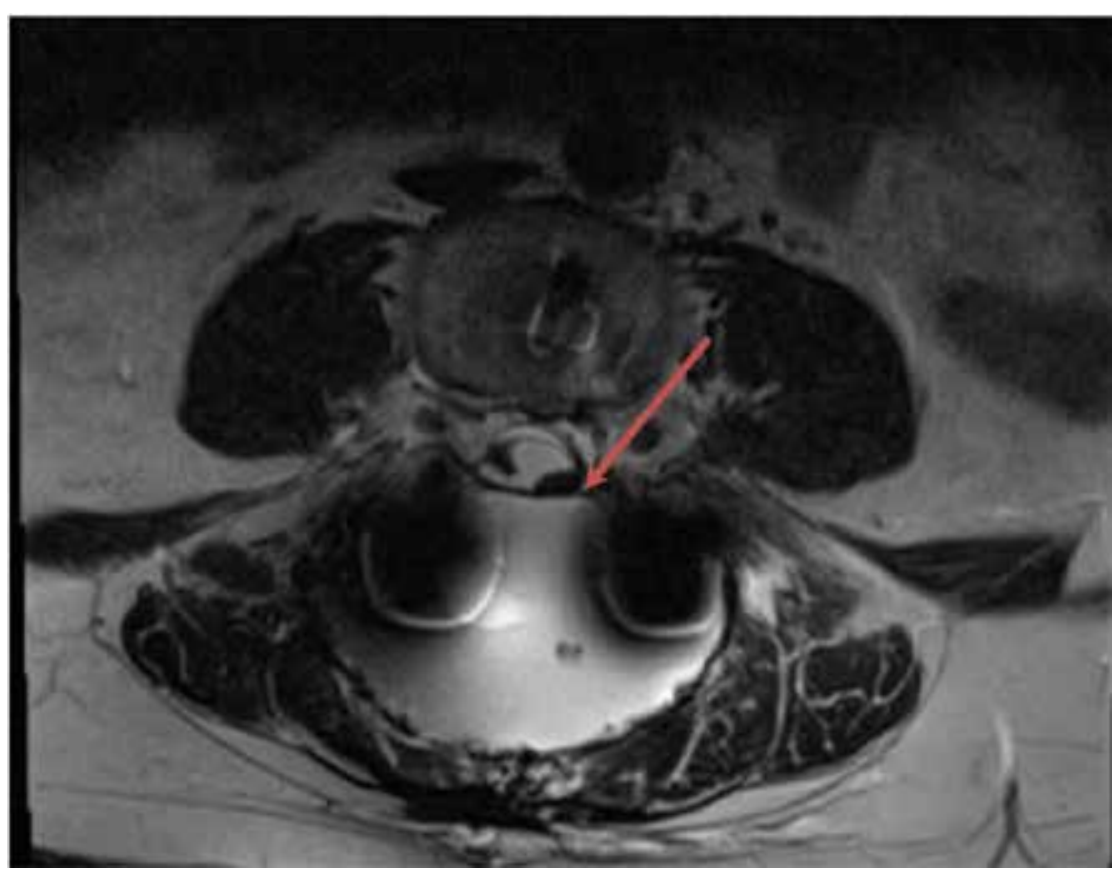

Fig. 2. Axial lumbar MRI demonstrates seroma formation (red arrow).

who undergo spinal surgeries or frequent interventional spinal procedures. Finally, this case reinforces the importance of reimaging patients when there is a history of trauma before considering routine interventional pain procedures.

\section{Consent}

Patient consent was obtained prior to publishing this case report.

\section{Acknowledgments}

The authors would like to thank the editors of Pain Medicine Case Reports for their guidance and assistance with this manuscript.

\section{REFERENCES}

1. Chan C, Peng P. Failed back surgery syndrome. Pain Med 2011; 12:577-606.

2. Akins PT, Harris J, Alvarez JL, et al. Risk factors associated with 30-day readmissions after instrumented spine surgery in 14,939 patients. Spine 2015; 40:1022-1032.

3. Cahill KS, Chi JH, Day A, Claus EB. Prevalence, complications, and hospital charges associated with use of bone-morphogenetic proteins in spinal fusion procedures. JAMA 2009; 302:58-66

4. Yew A, Kimball J, Lu DC. Surgical seroma formation following posterior cervical laminectomy and fusion without rhBMP-2: Case report. J Neurosurg Spine 2013; 19:297-300.

5. Epstein NE. More risks and complications for elective spine surgery in morbidly obese patients. Surg Neurol Int 2017; 8:66. 
